Research Article

\title{
Structure-Acoustic Coupling Analysis of Vibration and Underwater Acoustic Radiation of a Ring-Stiffened Conical Shell
}

\author{
Qingtao Gong, ${ }^{1}$ Zhanyang Chen $\mathbb{D}^{2},{ }^{2}$ Hongbin Gui, ${ }^{2}$ and Dong $\mathrm{Yu}^{2}$ \\ ${ }^{1}$ Ulshan Ship and Ocean College, Ludong Universtity, Yantai 264025, China \\ ${ }^{2}$ School of Ocean Engineering, Harbin Institute of Technology at Weihai, Weihai 264209, China \\ Correspondence should be addressed to Zhanyang Chen; chen_1228@163.com
}

Received 13 August 2021; Accepted 27 October 2021; Published 11 November 2021

Academic Editor: PengYao Yu

Copyright ( 2021 Qingtao Gong et al. This is an open access article distributed under the Creative Commons Attribution License, which permits unrestricted use, distribution, and reproduction in any medium, provided the original work is properly cited.

The underwater acoustic radiation of the submarine power cabin has recently become a hot topic in the industry and also in the academia. In this article, the vibration and underwater acoustic radiation of a ring-stiffened conical shell with bases are investigated numerically by means of the combination of the finite element method and boundary element method. The acoustic radiation field is obtained by the traditional acoustic field model and ISO acoustic field model, respectively. A series of numerical examples are given, and the results are compared. Besides, the sound pressure at different positions with frequency is further studied. It is shown that the sound radiated by the structure mainly propagates to the side directions of the shell and propagates relatively less to the front side and the rear side.

\section{Introduction}

Fluid-structure interaction of flexible structures is a common phenomenon in both engineering and natural environments $[1,2]$. In general, the surface vessels and submarines belong to flexible structure. The power cabin, which is located at the stern, directly determines the vibroacoustic characteristics and level of the whole ship. Consequently, structure-acoustic coupling analysis of the stern has great significance to the development of acoustic stealth. In physical applications, researchers and scholars usually regard the stern of submarine as a ring-stiffened conical shell and have conducted abundant research on the vibroacoustic problem [3-5].

The acoustic radiation is caused by the structural vibration. However, the structural vibration in the fluid medium is quite different from that in air or vacuum. Besides the vibration of structure itself, there is an interaction between the structure and fluid medium. When the structure is excited to produce vibration, the vibration boundary may compress the surrounding fluid to make the vibration propagate outwards in the form of wave, i.e., acoustic radiation. Meanwhile, the vibration of the surrounding fluid will occur and act on the structure as a radiation force, which is called the acoustic-structure coupling problem $[6,7]$. The underwater acoustic radiation of the submarine occurs in the complex environment with an interaction of the structural vibration and water medium. Zou et al. [1] pointed out that the interaction belongs to a strong coupling problem, which could not be neglected. Thus, for underwater acoustic radiation problems, the acoustic-structure coupling effect has to be considered.

Generally speaking, for some regularly shaped structures, such as plates, cylindrical shells, spherical shells, and so on, analytical solution to the acoustic-structure coupling problem is available, while for most elastic structures, numerical simulation is the only method for calculation of acoustic radiation induced by the vibrating surface of structures. Two basic numerical methods are used to deal with acoustic problems. One is the acoustic finite element method (FEM) $[8,9]$. The other one is the acoustic boundary element method (BEM) $[1,10,11]$. Liu et al. [12] studied the dynamic response and acoustic radiation of a blade-shafting-shell coupled system operating under unsteady excitation. Dynamic and acoustic experiments of the blade-shafting-shell coupled system were conducted. The results verified the accuracy of 
the simulation model. Qu et al. [13] investigated the structural and acoustic responses of a coupled propeller-shafting-submarine pressure hull system under different propeller force excitations. Finally, computed results were compared with those solutions obtained from the coupled FEM/BEM. The effects of the ring and the bearing stiffness on the acoustic responses of the coupled system were discussed. Zou et al. [14] established a calculation method of underwater acoustic radiation for three-dimensional structures arbitrarily covered by acoustic coatings based on the three-dimensional sonoelasticity theory for ships $[1,2]$. As an example, the acoustic radiation of a flat plate covered by acoustic coatings was numerically calculated and compared with analytical results, which verified the correctness of the proposed method. Moreover, a series of results for the cylindrical shell structure with partial acoustic coatings were presented, which showed the interest of this method in engineering practice.

Thus, the main purpose of this paper is to study the sound pressure level obtained by the different acoustic field models. By analyzing and quantifying the sound pressure level of different positions around the sound source, the spatial distribution law of acoustic radiation is presented, which will provide a reference for the acoustic stealth performance of submarine.

\section{Finite Element Model and Relevant Parameters}

2.1. Acoustic Finite Element Method. The FEM is widely used to investigate enclosure acoustic characteristics. According to the Helmholtz equation, the integral expression in the acoustic field can be written as

$$
\int_{V} \tilde{p}\left(\nabla^{2} p(x, y, z)-k^{2} p(x, y, z)+j \rho_{0} \omega q(x, y, z)\right) \mathrm{d} V=0 .
$$

After a series of analysis and formula, we have

$$
\int_{V}(\nabla \tilde{p} \cdot \nabla p) \mathrm{d} V-\omega^{2} \int_{V}\left(\frac{1}{c^{2}} \tilde{p} p\right) \mathrm{d} V=\int_{V} j \rho_{0} \omega \tilde{p} q \mathrm{~d} V-\int_{V}\left(j \rho_{0} \omega \widetilde{p} v \cdot n\right) \mathrm{d} \Omega .
$$

Equation (2) consists of four parts, and the first item on the left side can be expressed as

$$
\int_{V}(\nabla \tilde{p} \cdot \nabla p) \mathrm{d} V=\left\{\tilde{p}_{i}\right\}^{T} \cdot \int_{V}\left(\mathbf{B}^{T} \cdot \mathbf{B}\right) \mathrm{d} V \cdot\left\{p_{i}\right\}=\left\{\tilde{p}_{i}\right\}^{T} \cdot \mathbf{K} \cdot\left\{p_{i}\right\}
$$

where $\mathbf{K}$ is the stiffness matrix.

$$
K_{i j}=\int_{V}\left(B^{T} \cdot B\right) \mathrm{d} V=\int_{V}\left(\frac{\partial N_{i}}{\partial x} \cdot \frac{\partial N_{j}}{\partial x}+\frac{\partial N_{i}}{\partial y} \cdot \frac{\partial N_{j}}{\partial y}+\frac{\partial N_{i}}{\partial z} \cdot \frac{\partial N_{j}}{\partial z}\right) \mathrm{d} V
$$

The second item on the left side of equation (2) can be expressed as follows:

$$
-\omega^{2} \int_{V}\left(\frac{1}{c^{2}} \tilde{p} p\right) \mathrm{d} V=-\omega^{2}\left\{\widetilde{p}_{i}\right\}^{T} \cdot \mathbf{M} \cdot\left\{p_{i}\right\}
$$

where $\mathbf{M}$ is the mass matrix.

$$
M_{i j}=\int_{V}\left(\frac{1}{c^{2}} N_{i} N_{j}\right) \mathrm{d} V
$$

The first item on the right side of equation (2) can be expressed as follows:

$$
\int_{V} j \rho_{0} \omega \widetilde{p} q \mathrm{~d} V=\left\{\widetilde{p}_{i}\right\}^{T} \cdot\left\{Q_{i}\right\}
$$

where $\left\{Q_{i}\right\}$ is the acoustic excitation.

$$
\left\{Q_{i}\right\}=\int_{V}\left(j \rho_{0} \omega N^{T} q\right) \cdot \mathrm{d} V .
$$

The second item on the right side can be expressed as follows:

$$
-\int_{V}\left(j \rho_{0} \omega \tilde{p} v \cdot n\right) \mathrm{d} \Omega=-j \omega\left\{\widetilde{p}_{i}\right\}^{T} \cdot \mathbf{C} \cdot\left\{p_{i}\right\},
$$

where $\mathbf{C}$ is the damping matrix.

$$
C_{i j}=\int_{\Omega_{Z}}\left(\rho_{0} A N_{i} N_{j}\right) \mathrm{d} \Omega
$$

The dynamic equation of acoustic system will be obtained by substituting equations (4), (6), (8), and (10) into equation (2).

$$
\left(K+j \omega C-\omega^{2} M\right) \cdot\left\{p_{i}\right\}=\left\{Q_{i}\right\} .
$$

The Helmholtz solution in physical space will be obtained by solving the above equation.

2.2. Relevant Parameters. For the presented FEM/BEM scheme, LMS Virtual.Lab is applied to establish the FEM model and conduct the modal analysis. A ring-stiffened conical shell model with bases is built and shown in Figure 1(a), where steel is selected as the material, and density, Poisson's ratio, elastic modulus, and loss factor are $7800 \mathrm{~kg} / \mathrm{m}^{3}, 0.3,210 \mathrm{GPa}$, and 0.001 , respectively. The whole bases mainly consist of ribbed slabs, a plate, and a web, as shown in Figure 1(b). The main dimensions and relevant parameters are listed in Table 1.

The combination of FEM and BEM is used for the underwater structure-acoustic coupling analysis of the ring- 


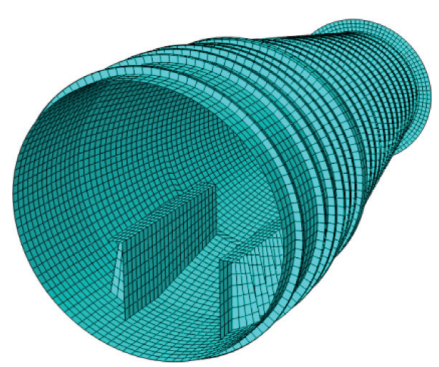

(a)

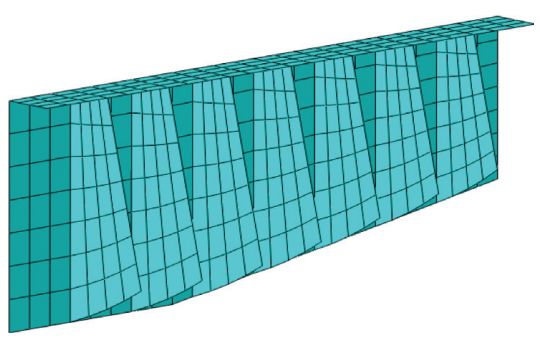

(b)

Figure 1: Finite element model. (a) Ring-stiffened conical shell. (b) Base.

Table 1: Main dimensions and relevant parameters.

\begin{tabular}{lc}
\hline Item & Value \\
\hline Full length (mm) & 3200 \\
Thickness (mm) & 7 \\
Diameter of the structural front-end (mm) & 1750 \\
Diameter of the structural back-end (mm) & 840 \\
Rib interval (mm) & 150 \\
Rib thickness (mm) & 5 \\
Plate thickness (mm) & 10 \\
Thickness of web (mm) & 5 \\
Thickness of ribbed slabs (mm) & 5 \\
Web width (mm) & 100 \\
Base length (mm) & 1200 \\
Distance of plate's front-end to shell surface (mm) & 370 \\
Distance of plate's back-end to shell surface (mm) & 532.5 \\
Distance from web to the longitudinal center plane of shell & 325 \\
(mm) &
\end{tabular}

stiffened conical shell in this study. The technical specification of the computing hardware used to conduct the numerical simulation in this paper is Intel Core i5-9400F CPU with 8 GB RAM. To investigate the coupled effect between the shell structure and external fluid medium, the finite element model of water $\left(\rho_{w}=1000 \mathrm{~kg} / \mathrm{m}^{3}\right)$ is built by the acoustic finite element tetrahedral meshes, the size of which is $10 \mathrm{~m} \times 10 \mathrm{~m} \times 10 \mathrm{~m}$, as shown in Figure 2. The middle part is removed for the shell structure. The conical shell that is located in the central part of the fluid medium is established by the structural finite element quadrilateral meshes. Besides, the inside of the conical shell is regarded as vacuum.

\section{Underwater Vibration and Acoustic Radiation of the Ring-Stiffened Conical Shell}

3.1. Vibration Modal Analysis in Vacuum. Prior to the acoustic radiation analysis, it is necessary to obtain the modes of the structure in vacuum. The results are presented in Figure 3. It is shown that the first two-order vibrations mainly occur on the shell, and the amplitudes of vibrations are small. Large radial vibration occurs only at the edge of the base web. Besides, the last six-order vibrations mainly occur at the base, which result from the vibrations of the web and ribbed slab, and the amplitudes are larger than those of first two-order vibrations.

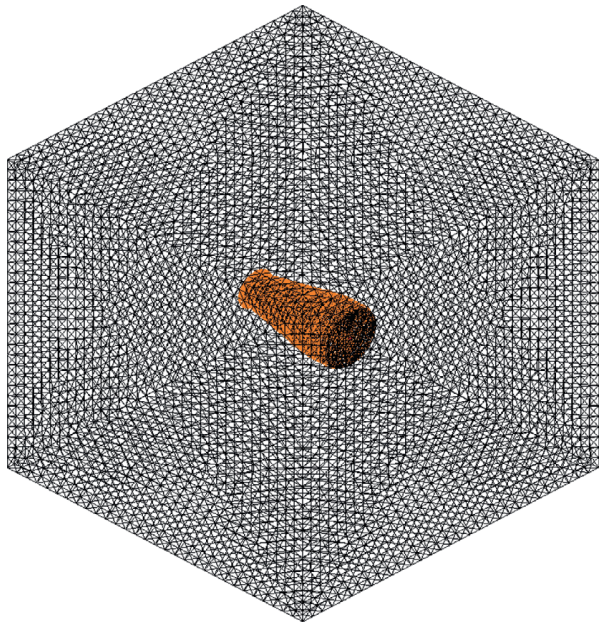

Figure 2: Fluid-structure coupled finite element model.

3.2. Establishment of Model for Acoustic Radiation. In this section, the acoustic analysis of the ring-stiffened conical shell system is conducted, and the acoustic radiation field of the structure at different frequencies is studied. The sinusoidal exciting force is imposed normally on the plate of the base with amplitude of $100 \mathrm{~Pa}$. The acoustic field model is built in the xoy and the yoz planes, respectively, the size of which is $20000 \mathrm{~mm}$ long $\times 20000 \mathrm{~mm}$ wide. The central axis of the conical shell coincides with the intersection of two fields. Besides, according to the ISO standard, the ISO acoustic field model is also established. The model is shown in Figure 4.

3.3. Results and Discussion from Different Acoustic Field Models. Due to the essence of FEM and restrictions of mesh, the frequency of $2970 \mathrm{~Hz}$ will reach the limit of the computer's calculation ability. Thus, the frequency range is from $50 \mathrm{~Hz}$ to $2950 \mathrm{~Hz}$, and the step is $50 \mathrm{~Hz}$. Figure 5 presents the distribution of sound pressure level $(\mathrm{dB})$ corresponding to different frequencies in the xoy plane. It is obvious that the acoustic radiation field takes a symmetrical distribution around the central axis in the xoy plane. The largest radiated sound pressure occurs in the areas on both sides of structure, and the sound pressure in front of the shell takes the second place, and the one behind the shell reaches the minimum. This trend becomes more and more obvious as the frequency 


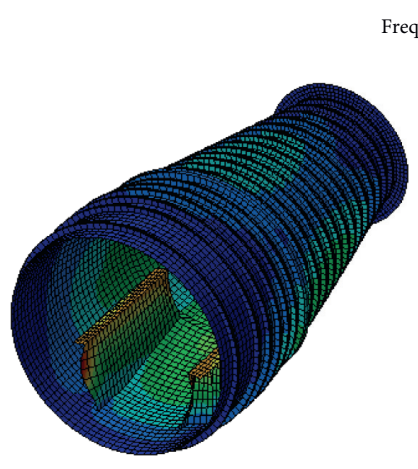

(a)



(c)

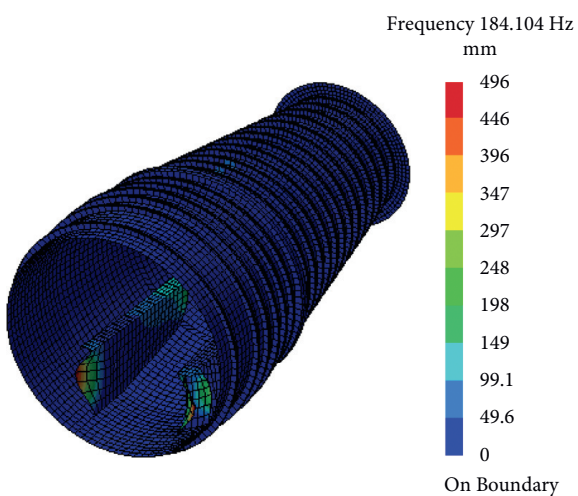

(e)

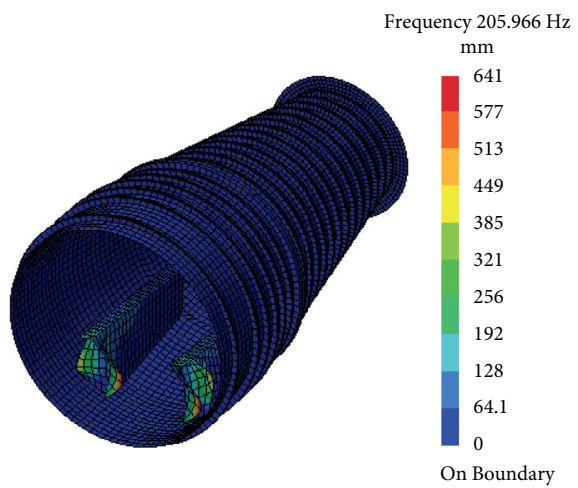

(g)

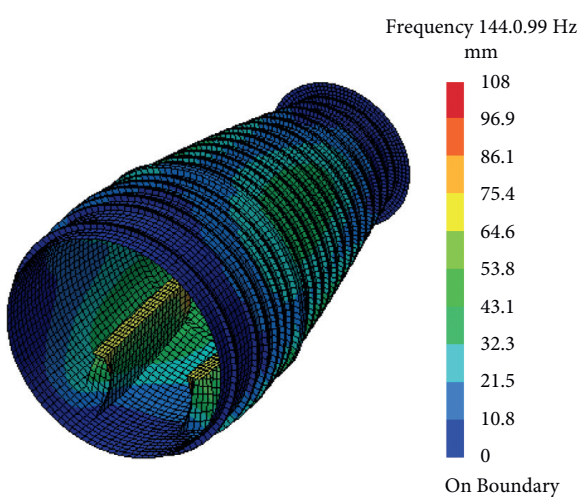

(b)



(d)

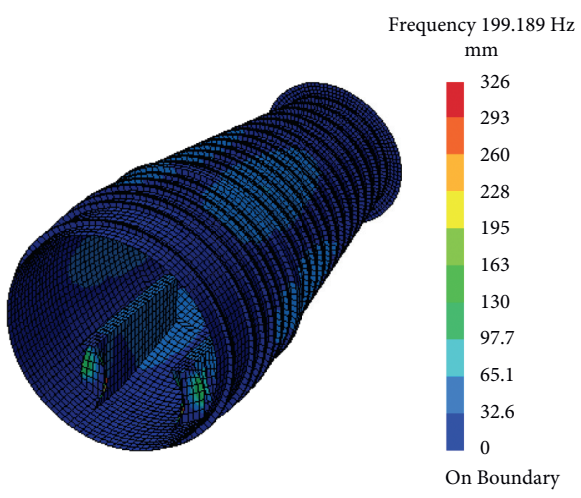

(f)



(h)

FIGURE 3: Modal shape of the ring-stiffened conical shell in vacuum. (a) The 1st order. (b) The 2nd order. (c) The 3rd order. (d) The 4th order. (e) The 5th order. (f) The 6th order. (g) The 7th order. (h) The 8th order. 


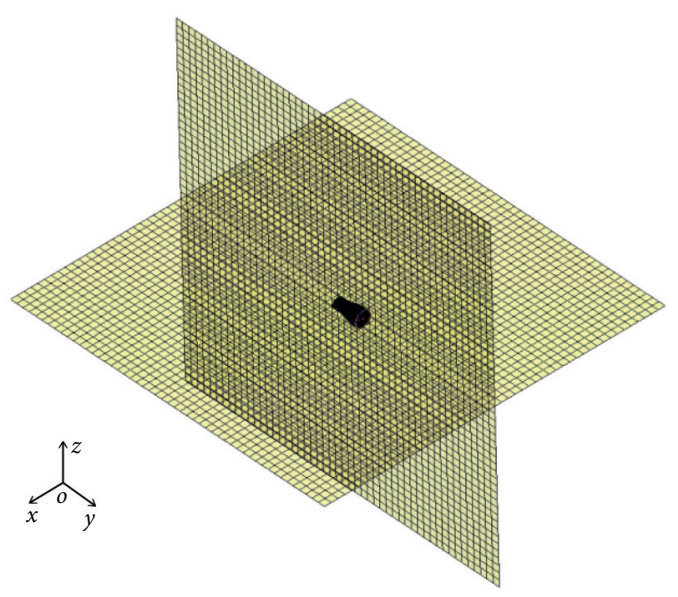

(a)



(b)

Figure 4: Models of acoustic field and structure. (a) Planar acoustic field model. (b) ISO acoustic field model.

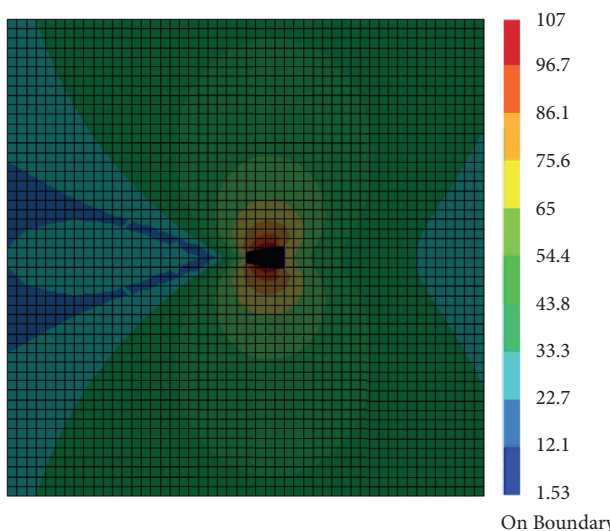

(a)

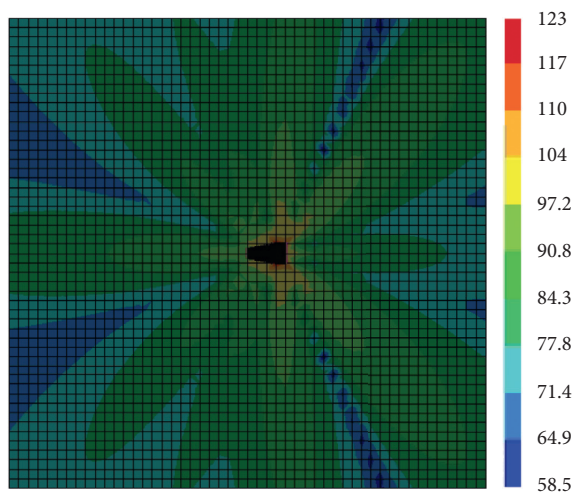

On Boundary

(c)

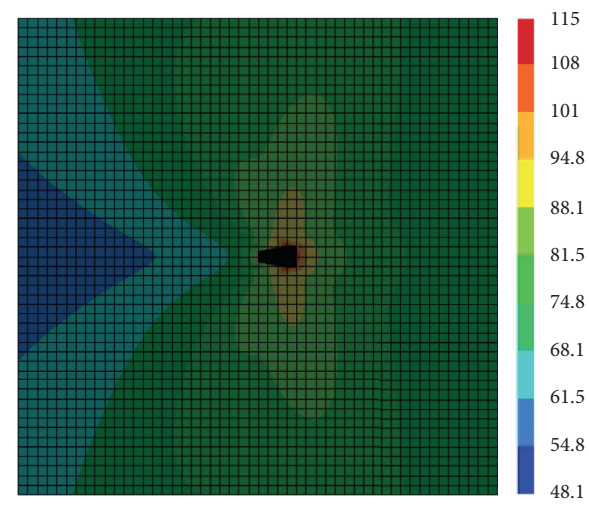

On Boundary

(b)

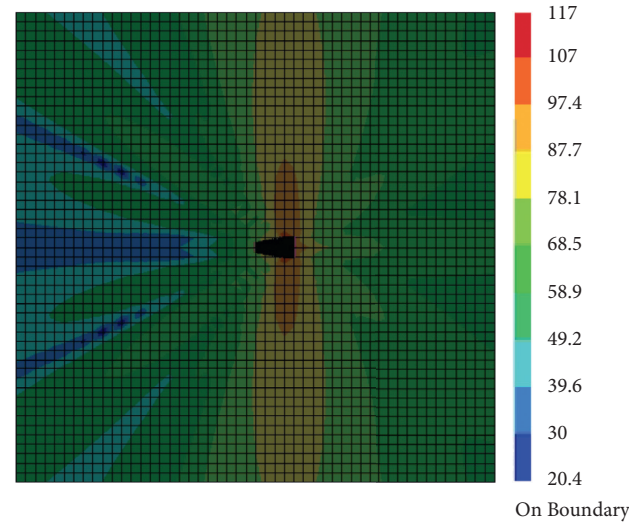

(d)

Figure 5: Acoustic radiation field at different frequencies in the $x o y$ plane. (a) $50 \mathrm{~Hz}$. (b) $1000 \mathrm{~Hz}$. (c) $2000 \mathrm{~Hz}$. (d) $2950 \mathrm{~Hz}$.

increases. Besides, the higher the frequency is, the more the lobes of the acoustic radiation field are and the more complex the waveform becomes. This indicates that the modal shapes become more complex as the frequency increases. The acoustic field radiated by the ring-stiffened conical shell mainly propagates to both sides and is closer to the bases.
Figure 6 presents the distribution of sound pressure level in the yoz plane. On the whole, the distribution in the yoz plane is similar to that in the xoy plane. However, since the source is on the bottom of the shell, the acoustic radiation field is not asymmetrical, and the sound pressure below the shell is greater than that above the shell. Compared with the sound pressure level in the xoy plane, the sound pressure 


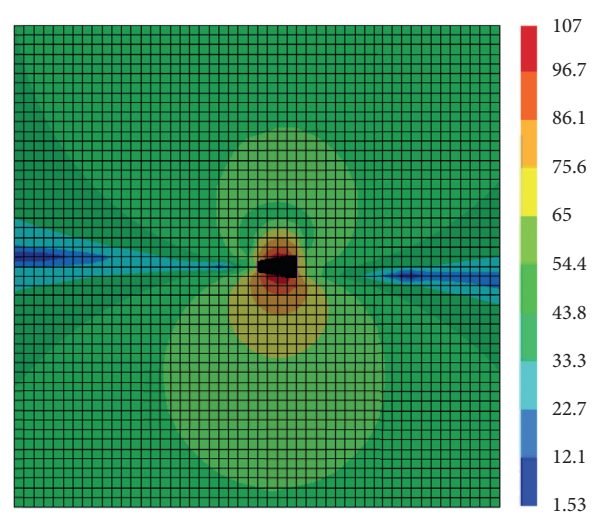

On Boundary

(a)

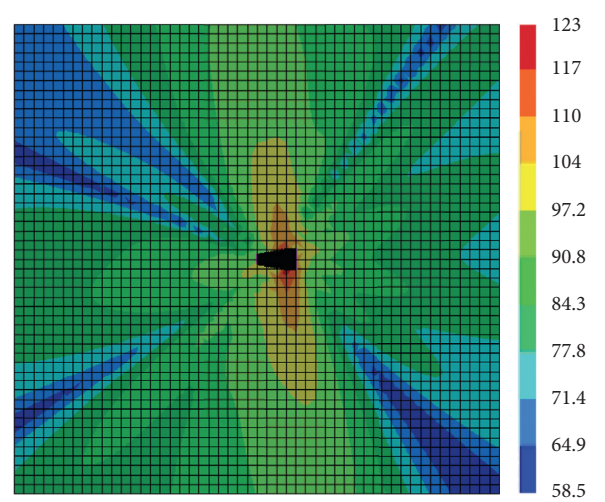

On Boundary

(c)

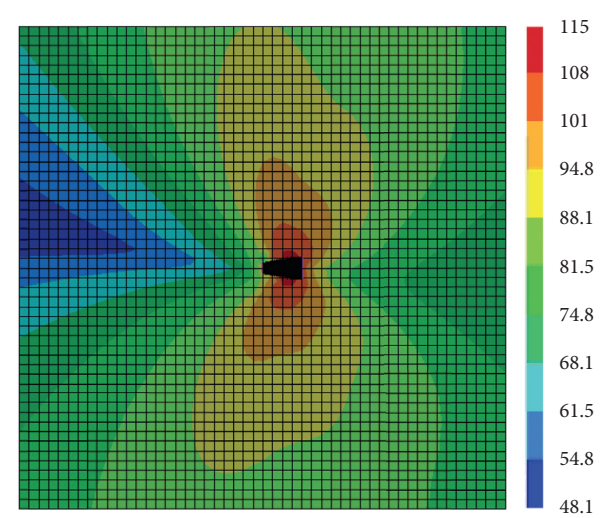

On Boundary

(b)



(d)

Figure 6: Acoustic radiation field at different frequencies in the yoz plane. (a) $50 \mathrm{~Hz}$. (b) $1000 \mathrm{~Hz}$. (c) $2000 \mathrm{~Hz}$. (d) $2950 \mathrm{~Hz}$.

below and above the shell is greater than that of each side at the same frequency. Thus, it can be seen that the sound radiated by the ring-stiffened conical shell travels further in both up and down directions.

In order to verify the conclusions above, the distribution of sound pressure level in the ISO acoustic field at different frequencies is calculated and presented in Figure 6, which shows the distribution of sound pressure at the same distance from the structure. The sound pressure below and above the shell is greater than that of other directions, and the sound pressure around the stern structure reaches the minimum. As discussed previously, the sound mainly spreads up and down, which has been further confirmed by Figure 7 .

3.4. Changes of Sound Pressure with Frequency and Positions. The changes of sound pressure with frequency in different positions are further studied, which can provide more convincing evidence. The six sample field points in both the xoy plane and the yoz plane are selected to analyze the sound pressure level, as shown in Figure 8. Figure 9 presents the sound pressure level of the six field points. Obviously, the change of sound pressure level with frequency is in a relatively stable state of fluctuation. The sound pressure of Point $c$ is the largest, and the sound pressure of Point $a$ takes the second place, and the sound pressure of Point $b$ is the lowest. So, we can conclude that the sound radiated by the shell mainly spreads to both sides, and the sound pressure in front of the shell is greater than that behind the shell in the xoy plane. Besides, although Point $f$ is closest to the shell, the sound pressure of Point $f$ is the minimum, while the sound pressure of Point $d$ and Point $e$ is almost the same. This also indicates that the sound pressure in both up and down directions is greater than that in front of the shell. 


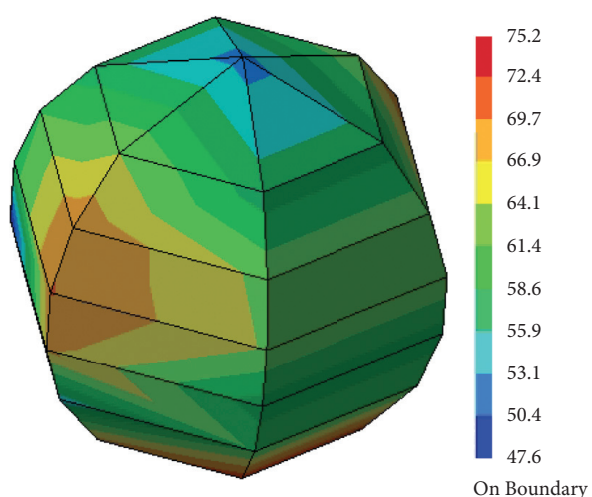

(a)

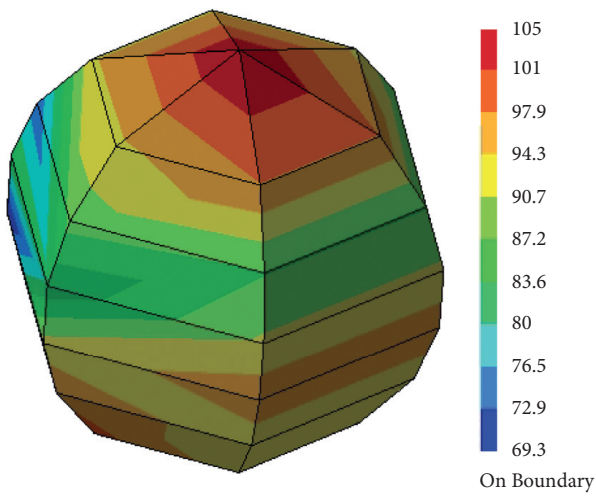

(c)

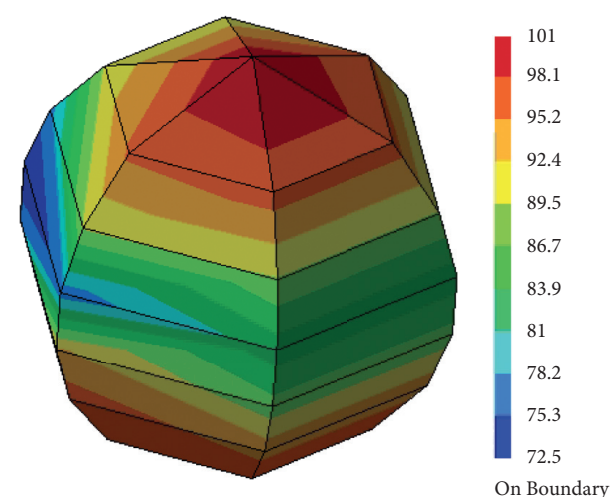

(b)

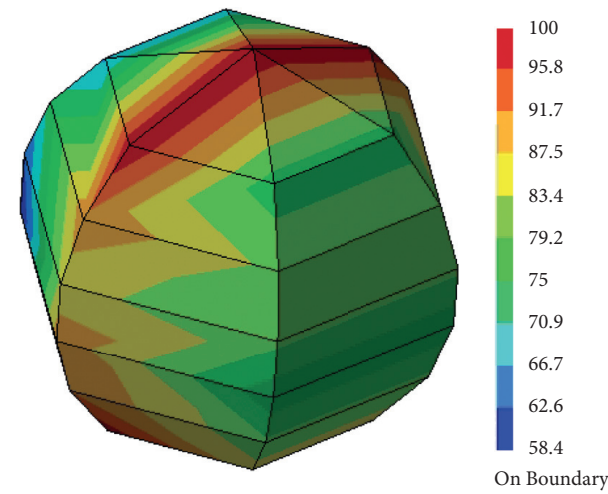

(d)

FIgURE 7: Acoustic radiation field at different frequencies based on ISO standard. (a) $50 \mathrm{~Hz}$. (b) $1000 \mathrm{~Hz}$. (c) $2000 \mathrm{~Hz}$. (d) $2950 \mathrm{~Hz}$.

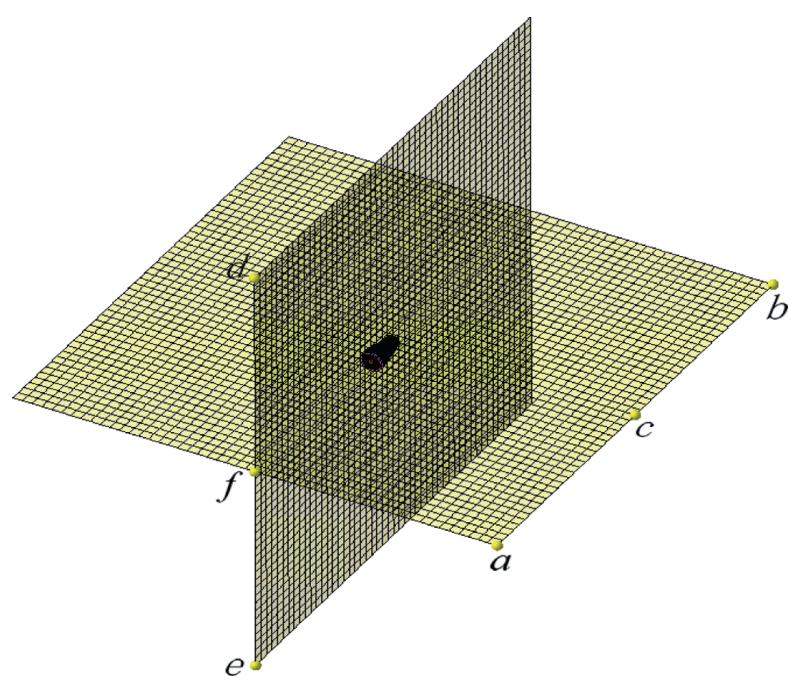

FIGURE 8: Six sample field points in the planar acoustic field. 


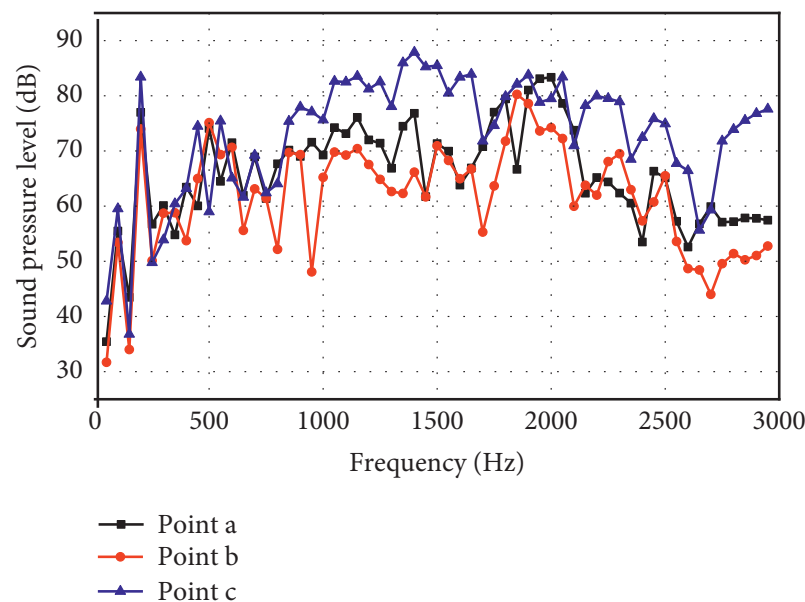

(a)

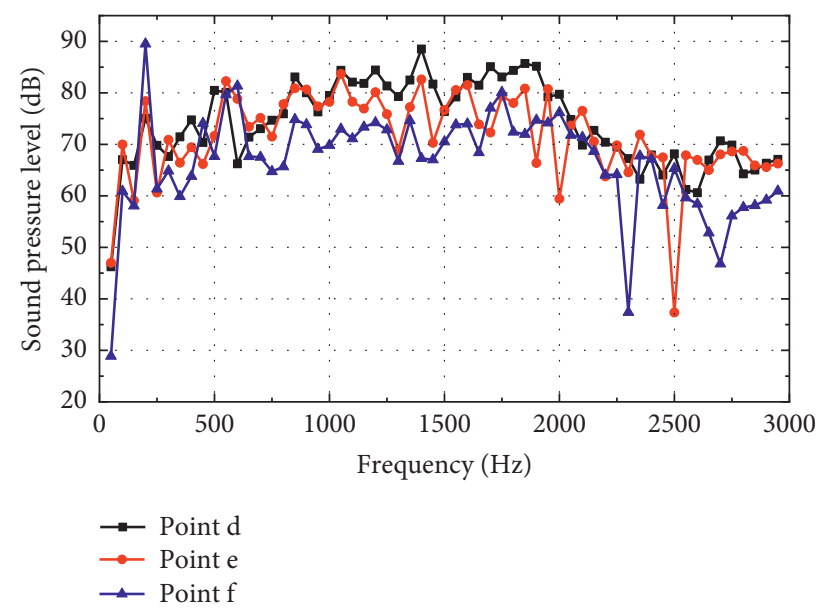

(b)

FIGURE 9: Sound pressure level of the six monitoring points with frequency. (a) In the xoy plane. (b) In the yoz plane.

\section{Conclusions}

A numerical analysis on the vibroacoustic responses of a typical ring-stiffened conical shell is carried out by using LMS Virtual.Lab. Some conclusions are drawn as follows:

(1) The first two-order vibrations mainly occur on the shell, and the amplitudes are small. The last six-order vibrations mainly occur at the base, which result from vibrations of web and ribbed slab, and the amplitudes are larger than those of first two-order vibrations.

(2) The distribution of sound pressure level obtained by the planar acoustic field model is the same as that of the ISO acoustic field model. The sound radiated by the structure mainly propagates to the side directions of the shell and propagates relatively less to the front side and the rear side. With the increase of the frequency, lobes of the acoustic radiation field become more and the waveforms become complex.

(3) Although some rules and conclusions obtained by this paper can provide an efficient tool for the analysis of the underwater acoustic radiation of the ring-stiffened conical shell, the physical model experiment is needed in the future for further validation.

\section{Data Availability}

All data, models, and codes generated or used during the study are available from the corresponding author upon request.

\section{Conflicts of Interest}

The authors declare that they have no conflicts of interest.

\section{Acknowledgments}

The authors acknowledge the financial support from the National Natural Science Foundation of China (grant no.
U2006229) and Key Research and Development Program of Shandong Province (grant nos. 2019JZZY010125 and 2020CXGC010701).

\section{References}

[1] M.-S. Zou, Y.-S. Wu, and S.-X. Liu, "A three-dimensional sono-elastic method of ships in finite depth water with experimental validation," Ocean Engineering, vol. 164, pp. 238-247, 2018.

[2] Y.-s. Wu, M.-s. Zou, C. Tian et al., "Theory and applications of coupled fluid-structure interactions of ships in waves and ocean acoustic environment," Journal of Hydrodynamics, vol. 28, no. 6, pp. 923-936, 2016.

[3] W. Guo, T. Li, X. Zhu, Y. Miao, and G. Zhang, "Vibration and acoustic radiation of a finite cylindrical shell submerged at finite depth from the free surface," Journal of Sound and Vibration, vol. 393, pp. 338-352, 2017.

[4] C. Wang and J. C. S. Lai, "The sound radiation efficiency of finite length circular cylindrical shells under mechanical excitation II: limitations of the infinite length model," Journal of Sound and Vibration, vol. 241, no. 5, pp. 825-838, 2001.

[5] M. Chen, D. Luo, and X. Chen, "Analytical solutions of radiation sound pressure of double cylindrical shells in fluid medium," Applied Mathematics and Mechanics, vol. 23, no. 4, pp. 463-470, 2002.

[6] W. Desmet, A Wave Based Prediction Technique for Coupled Vibro-Acoustic Analysis, Katholieke University Leuven, Belguim, 1998.

[7] B. Pluymers, W. Desmet, D. Vandepitte, and P. Sas, "Application of an efficient wave-based prediction technique for the analysis of vibro-acoustic radiation problems," Journal of Computational and Applied Mathematics, vol. 168, no. 1, pp. 353-364, 2004.

[8] L. Chen, H. Chen, C. Zheng, and S. Marburg, "Structuralacoustic sensitivity analysis of radiated sound power using a finite element/discontinuous fast multipole boundary element scheme," International Journal for Numerical Methods in Fluids, vol. 82, no. 12, pp. 858-878, 2016.

[9] C.-J. Zheng, C.-X. Bi, C. Zhang, H.-F. Gao, and H.-B. Chen, "Free vibration analysis of elastic structures submerged in an infinite or semi-infinite fluid domain by means of a coupled 
FE-BE solver," Journal of Computational Physics, vol. 359, pp. 183-198, 2018.

[10] L. W. Jiang, M. S. Zou, S. X Liu, and H. Huang, "Calculation method of acoustic radiation for floating bodies in shallow sea considering complex ocean acoustic environments," Journal of Sound and Vibration, vol. 476, 2020.

[11] S. Li and Q. Huang, "A fast multipole boundary element method based on the improved Burton-Miller formulation for three-dimensional acoustic problems," Engineering Analysis with Boundary Elements, vol. 35, no. 5, pp. 719-728, 2011.

[12] Y. Liu, J. Han, Z. Xue, Y. Zhang, and Q. Yang, "Structural vibrations and acoustic radiation of Blade-shafting-shell coupled system," Journal of Sound and Vibration, vol. 463, Article ID 114961, 2019.

[13] Y. Qu, J. Su, H. Hua, and G. Meng, "Structural vibration and acoustic radiation of coupled propeller-shafting and submarine hull system due to propeller forces," Journal of Sound and Vibration, vol. 401, pp. 76-93, 2017.

[14] M.-S. Zou, L.-W. Jiang, and S.-X. Liu, "Underwater acoustic radiation by structures arbitrarily covered with acoustic coatings," Journal of Sound and Vibration, vol. 443, pp. 748-763, 2019. 